\title{
Ecuaciones para la estimación de biomasa aérea y volumen de fuste de algunas especies leñosas nativas en el valle del río Foyel, NO de la Patagonia argentina
}

\author{
Equations for above-ground biomass and stem volume of some native woody species growing \\ in Foyel valley, NW Patagonia Argentina
}

\author{
Javier Gyenge ${ }^{\text {ab*, }}$, María Elena Fernández ${ }^{a b}$, Mauro Sarasola ${ }^{a}$, Mercedes de Urquiza ${ }^{a}$, Tomás Schlichter ${ }^{\mathrm{a}}$ \\ *Autor de correspondencia: aEstación Experimental Agropecuaria Bariloche INTA, CC 277, (8400) Bariloche, Río Negro, \\ Argentina, tel./fax: 54 (2944)422731, jgyenge@bariloche.inta.gov.ar \\ bConsejo Nacional de Investigaciones Científicas y Técnicas, CONICET, Argentina.
}

\begin{abstract}
SUMMARY
The forests and shrublands dominated by ñire (Nothofagus antarctica) represent one of the ecosystems with the highest latitudinal distribution within the Patagonian forests. They constitute most of the ecotone between the Andean forests and the Patagonian steppe. Forests and shrublands of ñire can be monospecific or be codominated by several species forming mixed systems with ñire, radal (Lomatia hirsuta), laura (Schinus patagonicus), and retamo (Diostea juncea). The objective of this publication is to present equations to predict total aboveground biomass (T) and their components: leaves, thin and thick branches, and stem. These estimations were obtained from easily measurable variables such as basal diameter of branches, and the stem, at breast height (DAP) or at stem base (DB) for each species. Also, we present equations to estimate stem volume up to $5 \mathrm{~cm}$ of diameter, DAP from DB and height from DAP. Between 6.5 to $14 \mathrm{~cm}$ of DAP, very few differences in $\mathrm{T}$ were observed among $\mathrm{T}$ of the four species. However, both ñire and radal showed trees with higher DAP and also, higher values of $\mathrm{T}$ compared to trees of laura and retamo.
\end{abstract}

Key words: biomass equations, ñire forests and shrublands.

\section{RESUMEN}

Los bosques y matorrales dominados por ñire (Nothofagus antarctica) se encuentran entre los ecosistemas de mayor distribución latitudinal dentro de los bosques andino patagónicos, conformando gran parte del ecotono bosque-estepa. En los matorrales mixtos las principales especies que acompañan al ñire son el radal (Lomatia hirsuta), la laura (Schinus patagonicus) y el retamo (Diostea juncea). El objetivo del trabajo fue el de ajustar una serie de ecuaciones que permitan estimar la biomasa aérea total (T) y la de sus componentes: hojas, ramas finas y gruesas y fuste, para las especies mencionadas. Dichas ecuaciones se obtuvieron para cada especie a partir de variables sencillas: diámetro basal de rama y fuste, diámetro a la altura del pecho (DAP) o en la base (DB). También se obtuvieron ecuaciones para estimar el volumen de fuste maderable hasta $5 \mathrm{~cm}$ de diámetro, el DAP a partir de DB, y la altura total a partir del DAP. En el rango de DAP de 6,5 a 14,0 cm no se observaron diferencias notables en T de las distintas especies. Sin embargo, como los individuos de ñire y de radal superaron dichos tamaños de DAP, mostraron mayores $\mathrm{T}$ que las restantes dos especies.

Palabras clave: ecuaciones de biomasa, bosques y matorrales de ñire.

\section{INTRODUCCIÓN}

Los bosques constituyen uno de los principales reservorios de carbono. Por lo tanto, en términos de la mitigación del cambio climático, es importante determinar la capacidad de un sistema boscoso para fijar carbono en todos sus componentes. Existen algunos antecedentes en la región patagónica de Argentina en los que se cuantificó la acumulación de carbono almacenado en biomasa y en suelo de distintos sistemas, habiéndose encontrado diferencias en el carbono acumulado en la vegetación de bosques de ciprés de la cordillera (Austrocedrus chilensis (D. Don.) Pic. Serm. et Biz.), plantaciones de pino ponderosa (Pinus ponderosa Douglas ex Lawson et Lawson) y la estepa graminosa de coirón dulce (Festuca pallescens (St. Yves) Parodi) (Laclau 2003). En estos mismos sistemas no se encontraron diferencias en el carbono secuestrado en el suelo al comparar los dos tipos de bosque evaluados (Laclau 2003). Además del secuestro de carbono, desde el punto de vista económico, también es importante determinar el volumen de madera en un sistema leñoso aprovechable, para así poder establecer distintas opciones de manejo del mismo (por ejemplo, manejo silvopastoril, extracción de leña, etc.; Reque et al. 2007). Por ello, tanto desde el punto de vista de los servicios ambientales como de bienes maderables es importante contar con ecuaciones 
que permitan estimar la biomasa de árboles y arbustos a partir de mediciones sencillas, como el diámetro a una determinada altura del fuste.

En general, son escasas las publicaciones de modelos que permitan estimar la biomasa o el volumen de fuste de especies leñosas nativas de la Patagonia argentina, centrándose en las especies de mayor valor maderero (en particular el ñire, Nothofagus antarctica (Forst.) Oerst. y ciprés de la cordillera). Los bosques y matorrales de ñire son algunos de los ecosistemas boscosos de mayor distribución latitudinal dentro de los bosques andino patagónicos y en gran parte constituyen el ecotono bosque-estepa (Vidal y Premoli 2004). El ñire se encuentra formando bosques monoespecíficos y matorrales mixtos. En los matorrales mixtos las principales especies arbóreas que lo acompañan son el radal (Lomatia hirsuta (Lam.) Diels ex J.F. Macbr.), la laura (Schinus patagonicus (Phil.) I. M. Johnst. ex Cabrera) y el retamo (Diostea juncea (Gillies ex Hook.) Miers (Schlichter y Laclau 1998, Reque et al. 2007). Reque et al. (2007) demostraron la potencialidad de estos matorrales mixtos de producir madera (principalmente para postes y leña), a pesar de la marcada variabilidad y heterogeneidad espacial. En este sentido, Sarasola et al. (2008) mostraron que, tras la aplicación de raleos, el crecimiento radial anual de árboles de ñire se incrementó en promedio hasta un $200 \%$. En relación a las especies mencionadas, se pueden nombrar trabajos en los cuales se muestran ecuaciones alométricas para ñire (Martínez Pastur et al. 1995, Lencinas et al. 2002, Peri et al. 2006, Gargaglione et al. 2008), llevados a cabo en la zona sur de Patagonia y en la Isla de Tierra del Fuego. Es importante destacar el trabajo de Laclau (1983) en el que determinó una ecuación para ñire que permite estimar el volumen de árbol que más se aproxima a las características promedio del rodal ("árbol tipo") para la provincia de Neuquén, Argentina ( $71^{\circ} 40^{\prime} \mathrm{O}$ y $40^{\circ} 09^{\prime} \mathrm{S}$ ).

Este trabajo tiene como fin principal aportar ecuaciones que, a partir de mediciones sencillas (diámetro a la altura del pecho o a la base de la planta), permitan estimar el peso seco de distintos compartimentos (hojas, ramas finas, ramas gruesas y fuste) de individuos de las principales especies que crecen en el matorral y bosque bajo de ñire, en el valle del río Foyel, NO de la Patagonia. Asimismo, con el objetivo de resumir la información generada en este tipo de formación boscosa, se incluyen en el trabajo ecuaciones publicadas que relacionan variables de interés biológico y forestal con el ánimo de completar un listado de ecuaciones que permitan estimar biomasa, volumen, o de forma de fuste y árbol. De esta manera, se brindan herramientas que permiten dar valor a las formaciones de matorral mixto desde aspectos comerciales, biológicos y de servicios ambientales. Este trabajo es parte de un proyecto mayor que tuvo por objeto determinar el impacto ambiental y productivo de distintos usos y reemplazos del matorral y bosque de ñire. Se espera que este trabajo constituya uno de los primeros antecedentes que permitan estimar la biomasa de algunas especies leñosas en el NO de la Patagonia.

\section{MÉTODOS}

El área de estudio abarcó ambientes dominados por ñire en la cuenca del río Foyel, tributario del río Manso (provincia de Río Negro, Argentina), y ocupa aproximadamente 100.000 ha con su centro geográfico en la

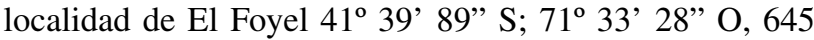
m s.n.m. (figura 1).

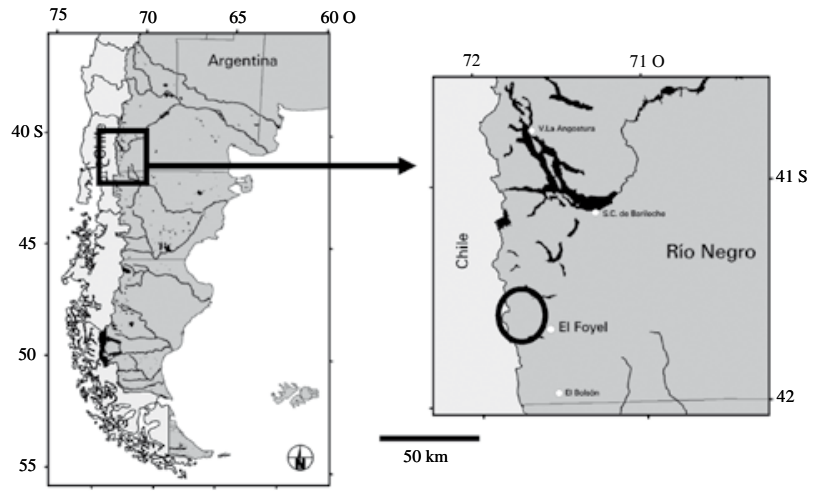

Figura 1. Localización del área de estudio. El círculo en el mapa de la derecha muestra la localización del sitio donde se realizó el apeo de los árboles (gentileza F. Umaña, Lab. Teledetección EEA Bariloche INTA).

Location of the study site. Circle in the right-side map shows the location in which trees were felled (map kindly provided by F. Umaña, Lab. Teledetección EEA Bariloche INTA).

Estudios en la zona determinaron que las especies leñosas dominantes son laura, ñire, radal y retamo (Reque et al. 2007, Gyenge et al. 2008). La temperatura media, precipitación y evapotranspiración anual del sitio (periodo 1969-1977) fueron de 7,3 ${ }^{\circ} \mathrm{C}, 1.414,3$ y $560 \mathrm{~mm}$, respectivamente (Gallopin 1978). El suelo, proveniente de cenizas volcánicas, mostró una alta capacidad de retención de humedad, baja densidad aparente $\left(0,8 \mathrm{~g} \mathrm{~cm}^{-3}\right)$, alto contenido de materia orgánica (6-8\%) y una textura franca a franco-limosa. La profundidad efectiva es moderada dado que a los $60 \mathrm{~cm}$ se observó la presencia de un sedimento de origen glacifluvial de textura arcillosa y de muy baja permeabilidad que se evidencia por la presencia muy abundante de moteados de hierro y colores gleyzados (Gyenge et al. 2008). La abundancia de cada especie fue muy variable dependiendo de la historia de uso del sitio en particular. Para el sitio de estudio se determinó que aproximadamente el $80 \%$ del área basal total estuvo representada por las cuatro especies mencionadas, con una densidad promedio total de individuos vivos de $6.618 \pm$ 3.349 árboles (media \pm desvío estándar, Gyenge et al. 2008; Fernández et al. 2009). Del total de fustes presentes, entre 
un 10 y $30 \%$ de ellos estaba muerto. La distribución de DAP de los árboles apeados fue de: laura 3,7 a 12,8 cm; ñire 6,8 a $21,1 \mathrm{~cm}$; radal 4,8 a $25,4 \mathrm{~cm}$ y retamo 5,5 a $15,2 \mathrm{~cm}$. Las $\mathrm{H}$ máximas y mínimas por especie fueron de: laura 3,7 y $6,2 \mathrm{~m}$; ñire 4,6 y $9,8 \mathrm{~m}$; radal 5 y 8,2 $\mathrm{m}$ y retamo 4,2 y $8,1 \mathrm{~m}$. En cuanto a los diámetros de copa, los mínimos y máximos diámetros medidos fueron: laura 1,0 y 2,9 m; ñire 0,75 y 4,6 m; radal 1,3 y 4,0 m y retamo 0,8 a $2,8 \mathrm{~m}$.

Las ecuaciones que permiten estimar la biomasa se formularon a partir del apeo de un número variable de individuos de ñire $(\mathrm{n}=16)$, radal $(\mathrm{n}=8)$, retamo $(\mathrm{n}=10)$ y laura $(n=8)$. Se definió como individuo aquel árbol cuyo eje principal no presentaba bifurcaciones por debajo de la medición de DAP (1,30 m de altura, Cailliez 1980). Se distribuyeron parcelas al azar dentro del rodal en donde se apearon los individuos a medir. Si bien el sitio de corta se seleccionó al azar, el número de individuos medidos de cada especie fue repartido uniformemente entre las diferentes clases diamétricas. Antes de ser apeado, al árbol se le midieron dos diámetros ortogonales de la copa (D1 y D2, $\mathrm{cm})$, la altura total $(\mathrm{H}, \mathrm{m})$, los diámetros del fuste en la base (a $15 \mathrm{~cm}$ de la superficie del suelo, DB, cm) y a la altura del pecho (DAP, cm). Los árboles fueron cubicados según la fórmula de Smalian (Cailliez 1980) con longitud de troza igual o menor a un metro. Cuando el fuste presentó marcadas variaciones morfológicas (horquillas, fendas, cambios de eje central, etc.), la longitud de las trozas fue definida por la longitud de las de formas más uniformes, siempre menores a $100 \mathrm{~cm}$. El diámetro mínimo inventariable (medido a $15 \mathrm{~cm}$ del suelo) se estableció en $3 \mathrm{~cm}$. Se consideró como fuste aquella porción de tronco con diámetro mayor a $5 \mathrm{~cm}$. Para cada árbol apeado se midió el diámetro de todas las ramas gruesas (aquellas con diámetros mayores a $5 \mathrm{~cm}$, DIAM cm). Además, de cada árbol se separaron al menos tres ramas gruesas a las cuales se les midió el peso fresco de los compartimentos: rama gruesa, ramas finas (aquellas con hojas) y hojas. Se pesaron todas las trozas de fuste, llevándose al laboratorio una o dos de ellas (dependido de la altura del fuste) para ser secadas. En el caso particular del retamo, el compartimento ramas finas incluyó a las hojas dado que las bases foliares son envainadoras. Todo el material extraído fue llevado a laboratorio donde fue secado en estufa a $70^{\circ} \mathrm{C}$ hasta lograr un peso constante. Una vez seco, se pesó el material definiéndose las siguientes variables: peso seco de hojas ( $\mathrm{Hs}, \mathrm{kg}$ ), peso seco de ramas finas $(\mathrm{Rm}, \mathrm{kg}) \mathrm{y}$ peso seco de ramas gruesas $(\mathrm{Rg}, \mathrm{kg})$.

A partir de los valores de peso seco de las ramas extraídas, se relacionaron mediante análisis de regresión lineal las variables $\mathrm{Hs}$, $\mathrm{Rm}$ y $\mathrm{Rg}$ (variables dependientes) con DB, DAP y DIAM (variables independientes). La validez de los modelos de regresión fue verificada a través de ANOVA, habiendo analizado los supuestos de normalidad (prueba de Kolmogorov-Smirnov) y homocedasticidad (correlación de Spearman; Dytham 2003). En caso de incumplimiento de los supuestos, se transformaron las variables hasta poder superarlos. Una vez determinadas las ecuaciones, se estimó la biomasa total de cada árbol sumando los valores de $\mathrm{Hs}, \mathrm{Rm}$ y $\mathrm{Rg}$ para cada rama. Las variables dependientes: biomasa en peso seco del fuste con corteza $(\mathrm{F}, \mathrm{kg})$, biomasa aérea total $(\mathrm{T}, \mathrm{kg})$ y el volumen del fuste con corteza hasta los $5 \mathrm{~cm}$ de diámetro (VOL, $\mathrm{m}^{3}$ ) se relacionaron con las variables independientes altura, y diámetros de fuste, ramas y copa mediante análisis de regresión múltiple (método: por pasos; probabilidad de $\mathrm{F}$ para entrar $\leq 0,05$; probabilidad de $\mathrm{F}$ para salir $\geq 0,05$ ).

\section{RESULTADOS}

Los modelos obtenidos permiten estimar, con distintos grados de precisión, los componentes de $\mathrm{Hs}, \mathrm{Rm}$ y $\mathrm{Rg}$ a partir de DIAM. Asimismo, los análisis de regresión múltiple demostraron que las variables que explican en mayor grado la variación del peso seco de los distintos componentes de la parte aérea del árbol dependieron de la especie (cuadros 1 y 2). En el caso de la laura, las ecuaciones desarrolladas teniendo el DAP como variable independiente mostraron los mayores ajustes, con excepción de la ecuación que permite estimar el peso seco de $\mathrm{Rg}$. En el caso del ñire, se observaron mayores ajustes en Rm y Rg utilizando el DIAM como variable independiente, mientras que en las ecuaciones para estimar Hs, F, VOL y T el mayor ajuste se obtuvo utilizando DAP como variable independiente (cuadro 1). En el caso del radal (cuadro 2) no se observó un patrón general, lográndose los mayores ajustes con las variables independientes DIAM (para Hs y $\mathrm{Rg}$ ), DB (para Rm) y DAP (F, VOL y T). Por último, se observó que en la mayor cantidad de ecuaciones, el mayor ajuste se obtuvo utilizando DB como variable independiente (excepto para estimar VOL, cuadro 2). Por otro lado, se encontró una alta relación entre DAP y DB, observándose una mayor variabilidad en la relación entre H y DAP, en cuyo caso, el ajuste de la regresión varió entre 0,44 y 0,62 (cuadro 3 ).

\section{DISCUSIÓN}

En general, son escasas las ecuaciones de biomasa de las especies leñosas nativas de la Patagonia, no encontrándose publicaciones con ecuaciones para laura, retamo y radal. Es importante señalar que la validez de las ecuaciones generadas en este estudio debe restringirse al rango de variación de las dimensiones del arbolado incluido en la muestra y la zona geográfica. Su extrapolación sólo debe hacerse a las mismas especies en otros sitios o regiones siempre y cuando presenten características similares de crecimiento.

La comparación de estimaciones por distintos modelos sólo puede ser realizada para ñire, dado que sólo existe 
Cuadro 1. Listado de ecuaciones y sus estadísticos que relacionan las variables dependientes: peso seco de hojas (Hs), ramas finas $(\mathrm{Rm})$, ramas gruesas $(\mathrm{Rg})$, peso seco y volumen de fuste (F y VOL, respectivamente) y peso seco de la biomasa aérea ( $\mathrm{T}$ ), con las variables independientes: diámetro de la rama (DIAM), a la altura del pecho (DAP) o a la base (DB) para individuos de laura (Schinus patagonicus) y ñire (Nothofagus antarctica).

Equations and their statistical parameters for the relationship between the dependent variables: dry weight of leaves (Hs), thin branches $(\mathrm{Rm})$, thick branches $(\mathrm{Rg})$, dry weight and volume of the stem (F and VOL, respectively) and aboveground dry weight (T), with the independent variables: diameter of the branch (diam), at breast height (DAP) or at the base of the stem (DB) for trees of laura (Schinus patagonicus) and nire (Nothofagus antarctica).

\begin{tabular}{|c|c|c|c|c|c|c|c|c|}
\hline \multirow{2}{*}{$\begin{array}{l}\text { Variable } \\
\text { dependiente }\end{array}$} & \multicolumn{4}{|c|}{ Laura } & \multicolumn{4}{|c|}{ Ñire } \\
\hline & Fórmula & $\mathrm{R}^{2}$ aj. & $\mathrm{n}$ & $P$ & Fórmula & $\mathrm{R}^{2}$ aj. & $\mathrm{n}$ & $P$ \\
\hline Hs & $-0,0431+0,0442 *$ DIAM & 0,67 & 26 & $* * *$ & $\mathrm{e}^{-4,443+0,638 * \text { DIAM }}$ & 0,65 & 44 & $* * *$ \\
\hline Hs & $-0,612+0,159 * \mathrm{DB}$ & 0,88 & 8 & $* * *$ & $\mathrm{e}^{-2,190+0,118 * \mathrm{DB}}$ & 0,72 & 14 & $* * *$ \\
\hline Hs & $-0,499+0,182 *$ DAP & 0,95 & 8 & $* * *$ & $-1,270+0,186 * \mathrm{DAP}$ & 0,75 & 8 & $* *$ \\
\hline $\mathrm{Rm}$ & $(0,0294+0,0710 * \text { DIAM })^{2}$ & 0,63 & 26 & $* * *$ & $\mathrm{e}^{-4,797+0,646 * \text { DIAM }}$ & 0,73 & 44 & $* * *$ \\
\hline $\mathrm{Rm}$ & $-0,368+0,138 *$ DAP & 0,93 & 8 & $* * *$ & $\mathrm{e}^{-2,537+0,119 * \mathrm{DB}}$ & 0,73 & 14 & $* * *$ \\
\hline $\mathrm{Rm}$ & $-0,452+0,120 * \mathrm{DB}$ & 0,86 & 8 & $* * *$ & $\mathrm{e}^{-2,798+0,182 * \text { DAP }}$ & 0,62 & 9 & $* * *$ \\
\hline $\mathrm{Rg}$ & $\mathrm{e}^{-3,787+0,890 * \text { DIAM }}$ & 0,77 & 22 & $* * *$ & $\mathrm{e}^{-3,382+2,256 * \ln (\mathrm{DIAM})}$ & 0,92 & 44 & $* * *$ \\
\hline $\operatorname{Rg}$ & $-6,948+1,595 * \mathrm{DB}$ & 0,66 & 8 & $* *$ & $-2,236+0,554 * \mathrm{DB}$ & 0,32 & 16 & $*$ \\
\hline $\mathrm{Rg}$ & $-6,086+1,865 * \mathrm{DAP}$ & 0,75 & 8 & $* *$ & $\mathrm{e}^{-1,424+0,209 * \mathrm{DAP}}$ & 0,60 & 10 & $* *$ \\
\hline $\mathrm{F}^{\mathrm{a}}$ & $\mathrm{e}^{-0,794+0,261 * \mathrm{DB}}$ & 0,91 & 8 & $* * *$ & $\mathrm{e}^{0,848+0,140 * \mathrm{DB}}$ & 0,75 & 16 & $* * *$ \\
\hline $\mathrm{F}^{\mathrm{a}}$ & $-8,950+2,314 * \mathrm{DAP}$ & 0,94 & 8 & $* * *$ & $\mathrm{e}^{0,263+0,206 * \mathrm{DAP}}$ & 0,89 & 10 & $* * *$ \\
\hline VOL & $-0,0113+0,00280 * \mathrm{DB}$ & 0,80 & 8 & $* * *$ & $(0,0169+0,0135 * \mathrm{DB})^{2}$ & 0,87 & 10 & $* * *$ \\
\hline $\mathrm{VOL}^{\mathrm{b}}$ & $(0,0106+0,0135 * \mathrm{DAP})^{2}$ & 0,91 & 8 & $* * *$ & $-0,0588+0,00884 * \mathrm{DAP}$ & 0,92 & 10 & $* * *$ \\
\hline $\mathrm{T}$ & $-18,025+3,852 * \mathrm{DB}$ & 0,82 & 8 & $* * *$ & $\mathrm{e}^{0,915+0,153 * \mathrm{DB}}$ & 0,82 & 16 & $* * *$ \\
\hline $\mathrm{T}$ & $-15,902+14,498 * \mathrm{DAP}$ & 0,92 & 8 & $* * *$ & $-53,873+7,373 *$ DAP & 0,94 & 10 & $* * *$ \\
\hline
\end{tabular}

$*=P<0,05 ; * *=P<0,01 ; * * *=P<0,001$. a Gyenge et al. (2008). ${ }^{b}$ Reque et al. (2007).

Cuadro 2. Listado de ecuaciones y sus estadísticos que relacionan las variables dependientes: peso seco de hojas (Hs), ramas finas $(\mathrm{Rm})$, ramas gruesas $(\mathrm{Rg})$, peso seco y volumen de fuste (F y VOL, respectivamente) y peso seco de la biomasa aérea ( $\mathrm{T}$ ), con las variables independientes: diámetro de la rama (DIAM), a la altura del pecho (DAP) o a la base (DB) para individuos de radal (Lomatia hirsuta) y retamo (Diostea juncea).

Equations and their statistical parameters for the relationship between the dependent variables: dry weight of leaves (Hs), thin branches $(\mathrm{Rm})$, thick branches $(\mathrm{Rg})$, dry weight and volume of the stem (F and VOL, respectively) and aboveground dry weight (T), with the independent variables: diameter of the branch (diam), at breast height (DAP) or at the base of the stem (DB) for trees of radal (Lomatia hirsuta) and retamo (Diostea juncea).

\begin{tabular}{|c|c|c|c|c|c|c|c|c|}
\hline \multirow{2}{*}{$\begin{array}{l}\text { Variable } \\
\text { dependiente }\end{array}$} & \multicolumn{4}{|c|}{ Radal } & \multicolumn{4}{|c|}{ Retamo } \\
\hline & Fórmula & $\mathrm{R}^{2}$ aj. & $\mathrm{n}$ & $P$ & Fórmula & $\mathrm{R}^{2}$ aj. & $\mathrm{n}$ & $P$ \\
\hline Hs & $\mathrm{e}^{-3,492+0,489 * \text { DIAM }}$ & 0,70 & 21 & $* * *$ & - & - & - & - \\
\hline $\mathrm{Hs}$ & $-2,279+0,448 * \mathrm{DB}$ & 0,56 & 8 & $*$ & - & - & - & - \\
\hline Hs & $-2,253+0,493 *$ DAP & 0,67 & 8 & $* *$ & - & - & - & - \\
\hline $\mathrm{Rm}$ & $\mathrm{e}^{-4,746+0,588 * \text { DIAM }}$ & 0,77 & 22 & $* * *$ & $\mathrm{e}^{-3,799+0,931 * \text { DIAM }}$ & 0,75 & 24 & $* * *$ \\
\hline $\mathrm{Rm}$ & $\mathrm{e}^{-2,180+0,144 * \mathrm{DB}}$ & 0,97 & 7 & $* * *$ & $-3,871+0,518 * \mathrm{DB}$ & 0,86 & 10 & $* * *$ \\
\hline $\mathrm{Rm}$ & $-1,380+0,225 * \mathrm{DAP}$ & 0,86 & 7 & $* *$ & $\mathrm{e}^{-2,888+0,312 * \mathrm{DAP}}$ & 0,71 & 10 & $* * *$ \\
\hline $\operatorname{Rg}$ & $(-0,299+0,343 * \text { DIAM })^{2}$ & 0,92 & 22 & $* * *$ & $\mathrm{e}^{-3,565+0,862^{*} \text { DIAM }}$ & 0,79 & 25 & $* * *$ \\
\hline $\mathrm{Rg}$ & $-12,412+1,954 * \mathrm{DB}$ & 0,68 & 8 & $* *$ & $-3,79+0,515 * \mathrm{DB}$ & 0,86 & 10 & $* * *$ \\
\hline $\mathrm{Rg}$ & $-11,771+2,109 * \mathrm{DAP}$ & 0,77 & 8 & $* *$ & $-2,988+0,517 * \mathrm{DAP}$ & 0,62 & 10 & $* *$ \\
\hline $\mathrm{F}^{\mathrm{a}}$ & $-31,047+4,062 * \mathrm{DB}$ & 0,82 & 8 & $* * *$ & $\mathrm{e}^{-0,446+0,236 * \mathrm{DB}}$ & 0,92 & 10 & $* * *$ \\
\hline $\mathrm{F}^{\mathrm{a}}$ & $-28,660+4,301 * \mathrm{DAP}$ & 0,88 & 8 & $* * *$ & $6,775+0,240 * \mathrm{DAP}$ & 0,80 & 10 & $* * *$ \\
\hline VOL & $-0,0501+0,00721 * \mathrm{DB}$ & 0,85 & 8 & $* * *$ & $-0,0282+0,00472 * \mathrm{DB}$ & 0,81 & 10 & $* * *$ \\
\hline $\mathrm{VOL}^{\mathrm{b}}$ & $-0,0467+0,00770 * \mathrm{DAP}$ & 0,93 & 8 & $* * *$ & $-0,0314+0,00573 * \mathrm{DAP}$ & 0,91 & 10 & $* * *$ \\
\hline $\mathrm{T}$ & $\mathrm{e}^{0,858+0,172 * \mathrm{DB}}$ & 0,86 & 8 & $* * *$ & $-28,936+4,126 * \mathrm{DB}$ & 0,92 & 10 & $* * *$ \\
\hline $\mathrm{T}$ & $-43,875+7,155 * \mathrm{DAP}$ & 0,91 & 8 & $* * *$ & $-25,556+4,428 *$ DAP & 0,79 & 10 & $* * *$ \\
\hline
\end{tabular}

$*=P<0,05 ; * *=P<0,01 ; * * *=P<0,001$. ${ }^{\mathrm{a}}$ Gyenge et al. (2008). ${ }^{\mathrm{b}}$ Reque et al. (2007). 
Cuadro 3. Ecuaciones y sus estadísticos que relacionan el diámetro a la altura del pecho (DAP) con el diámetro a la base (DB), y la altura total $(\mathrm{H})$ con el diámetro a la altura del pecho (DAP) para individuos de laura (Schinus patagonicus), ñire (Nothofagus antarctica), radal (Lomatia hirsuta) y retamo (Diostea juncea).

Equations and their statistical parameters for the relationship between the diameter at breast height (DAP) and the diameter at the base of the stem (DB) and between total height $(\mathrm{H})$ and diameter at breast height (DAP) for trees of laura (Schinus patagonicus), ñire (Nothofagus antarctica), radal (Lomatia hirsuta) and retamo (Diostea juncea).

\begin{tabular}{llrrc}
\hline \multicolumn{1}{c}{ Especie } & \multicolumn{1}{c}{ Fórmula } & $\mathrm{R}^{2}$ aj. & $\mathrm{n}$ & $P$ \\
\hline \multicolumn{5}{c}{ Variable dependiente: $\mathrm{DAP}$} \\
\hline Laura $^{\mathrm{a}}$ & $-0,524+0,862 * \mathrm{DB}$ & 0,90 & 8 & $* * *$ \\
Nire $^{\mathrm{a}}$ & $2,644+0,699 * \mathrm{DB}$ & 0,92 & 10 & $* * *$ \\
Radal & $\mathrm{e}^{1,360+0,0754 * \mathrm{DB}}$ & 0,93 & 8 & $* * *$ \\
Retamo & $-12,258+9,324 * \ln (\mathrm{DB})$ & 0,88 & 10 & $* * *$ \\
\hline \multicolumn{5}{c}{ Variable dependiente: $\mathrm{H}$} \\
\hline Laura $^{\mathrm{a}}$ & $3,236+0,195 *$ DAP & 0,44 & 8 & $*$ \\
Nire $^{\mathrm{a}}$ & $3,277+0,278 *$ DAP & 0,55 & 10 & $* *$ \\
Radal $^{\mathrm{a}}$ & $4,699+0,142 *$ DAP & 0,55 & 8 & $*$ \\
Retamo $^{\mathrm{a}}$ & $3,183+0,31 *$ DAP & 0,62 & 10 & $* *$ \\
\hline
\end{tabular}

$*=P<0,05 ; * *=P<0,01 ; * * *=P<0,001$. aReque et al. (2007).

información similar generada para esta especie. En este sentido, es posible comparar las predicciones de VOL en ñires en el NO de la Patagonia con ecuaciones locales de Tierra del Fuego desarrolladas en bosques con alturas dominantes de entre 8 y 10 metros (ecuación 1 en anexo, Lencinas et al. 2002). Para árboles con DAP similar, los ñires del NO de la Patagonia presentan menores volúmenes de fuste (figura 2). Sin embargo, al comparar las predicciones del modelo con una ecuación estándar tradicional (ecuación 2 en anexo, Lencinas et al. 2002) y la ecuación ajustada por Martínez Pastur et al. (1995; ecuación 3 en anexo) también para ñires de Tierra del Fuego, las predicciones son similares hasta un DAP $<18 \mathrm{~cm}(\mathrm{VOL}=0,10$ $\mathrm{m}^{3}$; figura 2). Es importante destacar que los DAP de los individuos de ñire en Tierra del Fuego duplican a los encontrados en el NO de la Patagonia. En este sentido, los DAP medidos en el NO de la Patagonia se corresponden con las fases de crecimiento óptimo inicial (DAP $=9,5 \pm$ error estándar $3 \mathrm{~cm}$ ) y final de ñires creciendo en Tierra del Fuego (DAP $=21,9 \pm 7,7 \mathrm{~cm}$ ) (Gargaglione et al. 2008). Es necesario destacar que, si bien se corresponden los tamaños de los individuos estudiados en Tierra del Fuego y los del presente estudio, no se determinó la edad de los ñires muestreados en el NO de la Patagonia, por lo que podría inducir un error el considerar que los individuos poseen edades similares. En este sentido, sería importante determinar la tasa de crecimiento por edades para las distintas condiciones de crecimiento en el NO de la Patagonia. Sin embargo, dependiendo del DAP, las predicciones indicarían que los ñires en el NO de la Patagonia tienen una mayor biomasa en la primera fase mencionada, mientras que presentan una menor biomasa en la fase de crecimiento óptimo final.

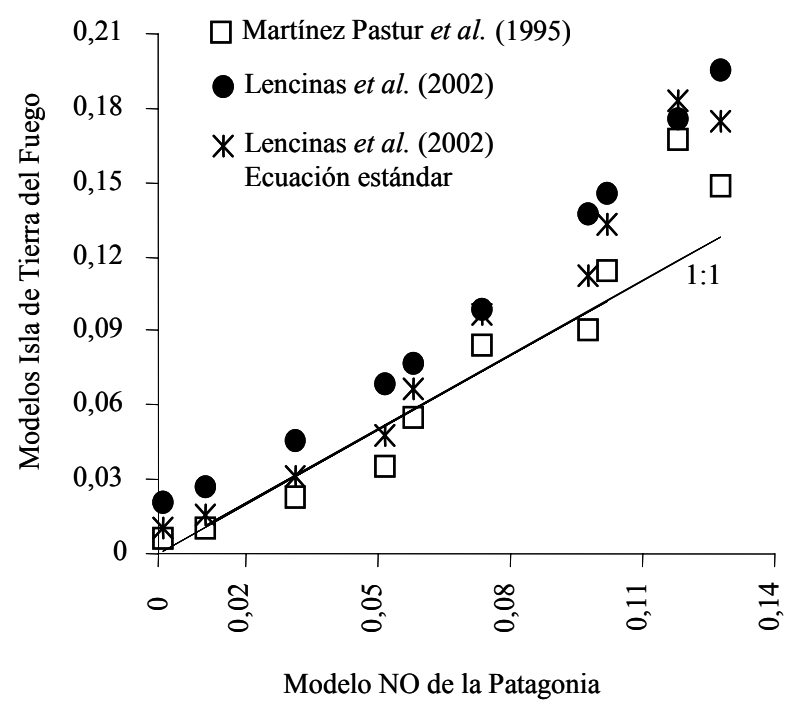

Figura 2. Relación entre las predicciones de volumen con corteza $\left(\mathrm{m}^{3}\right)$ del modelo generado en el NO de la Patagonia y los desarrollados para ñire (Nothofagus antarctica) en la Isla de Tierra del Fuego.

Relationship between stem volumes with bark $\left(\mathrm{m}^{3}\right)$ of ñire (Nothofagus antarctica) estimated using the model developed for NW Patagonia compared with those developed for Tierra del Fuego.

Si se comparan cualitativamente las predicciones de biomasa total aérea en relación a los diámetros de fuste de las cuatro especies estudiadas (en particular el DAP, figura 3), se observan, en general, pocas diferencias entre las mismas para el rango de tamaños de entre 6,5 y $14,0 \mathrm{~cm}$. Es importante destacar que se observaron mayores diámetros en los individuos de ñire y radal (aproximadamente 20 y $25 \mathrm{~cm}$, respectivamente) que en los individuos de laura y retamo (cuyos DAP máximos fueron de 12,8 y $15,2 \mathrm{~cm}$, respectivamente). Desde un punto de vista productivo, sería importante estudiar si los individuos de laura y retamo tienen capacidad de alcanzar mayores tamaños que los medidos. Sin embargo, dada la mayor tasa de crecimiento del ñire y radal (unas 10 veces mayor, Gyenge et al. 2008) y la posibilidad de que tanto el ñire como el radal alcancen mayores tamaños (más de $25 \mathrm{~cm}$ de diámetro), sería necesario plantear un manejo apropiado que permita maximizar la productividad de dichas especies. Por ejemplo, un mecanismo plausible podría ser el de extraer las especies de menor porte, o realizar raleos o floreos tendientes a bajar la densidad de individuos/fustes. En este sentido, Sarasola et al. (2008) comprobaron una alta respuesta en el crecimiento en ñires creciendo en rodales sometidos a raleos. Además, Gyenge et al. (2008) también mencionan que las especies de mayor productividad (en este caso, radal y ñire) poseerían una mayor eficiencia en el uso del agua que aquellas de 
menor productividad (kilogramo de madera por milímetro de agua consumida). De esta manera, extrayendo especies de baja productividad, se permitiría no sólo incrementar la tasa individual de crecimiento dándole un mayor valor de comercialización a ciertas especies nativas, sino también propender a optimizar el uso del agua.

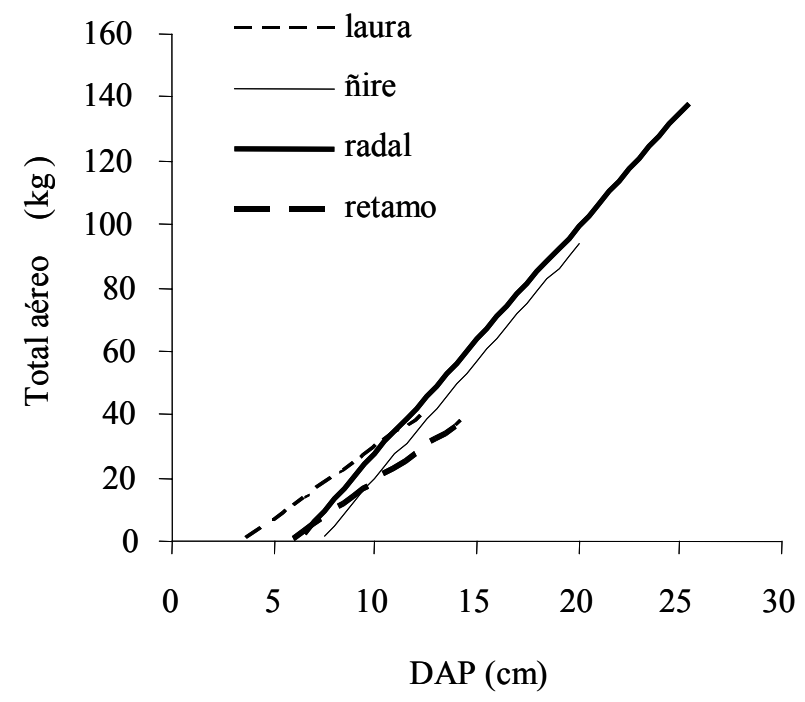

Figura 3. Relación entre la biomasa total aérea de árboles individuales en relación al diámetro a la altura del pecho (DAP) para las especies laura (Schinus patagonicus), ñire (Nothofagus antarctica), radal (Lomatia hirsuta) y retamo (Diostea juncea).

Relationship between total aboveground biomass of individual trees and diameter at breast height (DAP) for the species laura (Schinus patagonicus, $\mathrm{R}^{2} \mathrm{aj} .=0,92 ; P<0,001$ ), ñire (Nothofagus antarctica, $\mathrm{R}^{2}$ adj. $=0.94, P<0.001$ ), radal (Lomatia hirsuta, $\mathrm{R}^{2}$ adj. $=0.91, P<0.001$ ) and retamo (Diostea juncea, $\mathrm{R}^{2} \mathrm{adj} .=0.79, P<0.001$ ).

En cuanto a la capacidad de fijar carbono por parte del bosque mixto en la región del valle del río Foyel, Gyenge et al. (2008) demuestran que los mismos producen entre 737 y $4.760 \mathrm{~kg} / \mathrm{ha}$ de materia seca de fuste por año, dependiendo de la composición de especies y distribución de tamaños de los individuos. En los mismos sitios, los autores estimaron que la productividad anual de pino oregón (Pseudotsuga menziesii (Mirb) Franco) variaba de 2.964 a $7.909 \mathrm{~kg} / \mathrm{ha}$ de materia seca de fuste por año. Estas diferencias en productividad estarían basadas en que ambos tipos de vegetación difieren en su capacidad de regular su transpiración y, por ende, el flujo de carbono. En este sentido, las especies nativas demuestran, en general, una mayor resistencia hidráulica que la exótica (Fernández et al. 2009, Gyenge et al. 2009), lo que también produce una menor eficiencia en el uso del agua (Gyenge et al. 2008). Esto demostraría que, en promedio, es inferior la cantidad de carbono secuestrado en fuste por los bosques de matorrales mixtos en la región que por los bosques cultivados de pino oregón. Sin embargo, dependiendo del manejo de uno y otro, los valores podrían igualarse demostrando la importancia de los distintos tipos de intervenciones, tal como fue mencionado anteriormente.

Es importante destacar la necesidad de construir ecuaciones que permitan ampliar la capacidad predictiva con base en índices de sitio o clases de copa para los bosques de matorral mixto del NO de la Patagonia. La información brindada, de características locales, se torna valiosa dado el vacío de conocimiento sobre las especies arbóreas acompañantes del ñire en la mencionada zona, y permite avanzar en la valoración de los servicios ambientales y económicos de estos tipos de bosques.

\section{CONCLUSIONES}

Las ecuaciones presentadas permiten incrementar el conocimiento sobre producción y ecología de los bosques mixtos de la zona del valle del río Foyel. Asimismo, es necesario no sólo desarrollar modelos que estimen la biomasa, sino también aquellos que permitan estimar tasas de producción en respuesta a los distintos manejos y usos realizados en la zona: extracción de individuos muertos, raleos de distinta intensidad, uso silvopastoril, turísticos, etc.

\section{AGRADECIMIENTOS}

Este trabajo fue financiado a través de los proyectos Regional 61802 de INTA y PICT08-14692 (FONCYTMINCYT).

\section{REFERENCIAS}

Cailliez F. 1980. Estimación del volumen forestal y predicción del rendimiento con referencia especial a los trópicos. Vol. 1 - estimación del volumen. Estudio FAO 22/1. 80 p.

Dytham C. 2003. Choosing and using statistics, a biologist's guide, $2^{\mathrm{a}}$ Ed. Blackwell Publishing, Reino Unido. 248 p.

Fernández ME, JE Gyenge, T Schlichter. 2009. Water flux and canopy conductance of natural versus planted forests in Patagonia, South America. Trees 23: 415-427.

Gallopin GC. 1978. Estudio ecológico integrado de la cuenca del Río Manso superior (Río Negro, Argentina). Anales de Parques Nacionales XIV. p. 161-230.

Gargaglione V, PL Peri, G Martínez Pastur. 2008. Estimación de la biomasa aérea y subterránea de Nothofagus antarctica para diferentes clases de copa y edades. In EcoNothofagus 2008 - Segunda Reunión sobre Nothofagus en la Patagonia. Esquel, Chubut, Argentina. Libro de Actas. p. 131-136.

Gyenge JE, ME Fernández, M Sarasola, T Schlichter. 2008. Testing a hypothesis of the relationship between productivity and water use efficiency in Patagonian forests with native and exotic species. For. Ecol. Manage. 255: 3281-3287.

Gyenge JE, ME Fernández, T Schlichter. 2009. Effects on site water balance of conversion from native mixed forest to 
Douglas-fir plantation in N.W. Patagonia. New Forests DOI 10.1007/s11056-009-9132-0.

Laclau P. 1983. Estudio dendrométrico de una parcela implantada de "ñire" (Nothofagus antarctica) provincia de Neuquén, Argentina. Tesis Ingeniero Agrónomo. Buenos Aires, Argentina. Universidad de Buenos Aires. 31 p.

Laclau P. 2003. Biomass and carbon sequestration of ponderosa pine plantations and native cipress forests in Northwestern Patagonia. For. Ecol. Manage. 180: 317-333.

Lencinas MV, G Martínez Pastur, JM Cellini, R Vukasovic, P Peri, MC Fernández. 2002. Incorporación de la altura dominante y la clase de sitio a ecuaciones estándar de volumen para Nothofagus antarctica (Forster f.) Oersted. Bosque 23 (2): 5-17.

Martínez Pastur G, MC Fernández, F Boyeras, M Allogia. 1995. Estimación de la producción para ñire en Tierra del Fuego: 2. Ecuaciones estándar de volumen. In IV Jornadas Forestales Andino Patagónicas, San Martín de los Andes, Neuquén, Argentina. Libro de Actas. p. 145-150.

Peri PL, V Gargaglione, G Martínez Pastur. 2006. Dynamics of above- and below-ground biomass and nutrient accumulation in an age sequence of Nothofagus antarctica forest of Southern Patagonia . For. Ecol. Manage. 233: 85-99.

Reque J, M Sarasola, JE Gyenge, ME Fernández. 2007. Caracterización silvícola de ñirantales del norte de la Patagonia para la gestión forestal sostenible. Bosque 28 (1): 33-45.

Sarasola M, ME Fernández, J Gyenge, C Peyrou. 2008. Respuesta de los ñires al raleo en la cuenca del Río Foyel. In EcoNothofagus 2008 - Segunda Reunión sobre Nothofagus en la Patagonia. Esquel, Chubut, Argentina. Libro de Actas. p. 47.
Schlichter TM, P Laclau. 1998. Ecotono estepa-bosque y plantaciones forestales en la Patagonia norte. Ecología Austral 8: 285-296.

Vidal R, A Premoli. 2004. Variación en Nothofagus antarctica (Foster) Oerst. (ñirre o nire). In Donoso C, A Premoli, L Gallo, R Ipinza ed. Variación intraespecífica en las especies arbóreas de los bosques templados de Chile y Argentina. Santiago, Chile. Editorial Universitaria. p. 173-188.

\section{ANEXO}

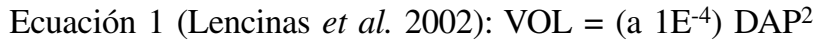
donde el coeficiente a es específico para cada sitio. En particular, se tomaron cuatro ecuaciones del trabajo de Lencinas et al. (2002) desarrolladas para rodales con altura dominante de entre 8 y $10 \mathrm{~m}$. Así, el valor de a fue de $5,06231448,3,63703298,4,94805662$ y 3,91269001 .

Ecuación 2 (Lencinas et al. 2002): VOL $=\left(0,59331 \mathrm{E}^{-4}\right)$ $\mathrm{DAP}^{2,16327} \mathrm{H}^{0,68172}$.

Ecuación 3 (Martínez Pastur et al. 1995): $\mathrm{VOL}=2,439 \mathrm{E}^{-05}$ $\mathrm{DAP}^{2,29752} \mathrm{H}^{0,71863}$.
Recibido: 24.11 .08

Aceptado: 24.04.09 